\title{
Quantification of myocardial extracellular volume fraction by cardiac magnetic resonance for early detection of the left ventricle involvement in systemic sclerosis
}

\author{
Daniel Lovrićc ${ }^{*}$, Franck Thuny², Claire Carmona', Frederic Schnell ${ }^{2}$, Laura Ernande ${ }^{2}$, \\ Helene Thibault ${ }^{2}$, Cyrille Bergerot ${ }^{2}$, Jacques Ninet ${ }^{3}$, Pierre Croisille ${ }^{4}$, Genevieve A. Derumeaux ${ }^{2}$ \\ ${ }^{1}$ Explorations Fonctionnelles Cardio-vasculaires, Louis Pradel Hospital, Lyon, France \\ ${ }^{2}$ Hospices Civils de Lyon, Université Claude Bernard Lyon, Lyon, France \\ ${ }^{3}$ Department of Internal Medicine, Edouard Herriot Hospital, Lyon, France \\ ${ }^{4}$ Creatis-LRMN, UMR CNRS 5220, INSERM U630, Lyon France
}

Background: Early identification of myocardial dysfunction is challenging for many cardiovascular diseases and systemic sclerosis (SSc) is an unique model of progressive myocardial fibrosis. Cardiac magnetic resonance with late gadolinium enhancement (LGE) can detect fibrosis in advanced stages of SSc, but is limited in the early stage due to the diffuse nature of fibrotic changes. T1 mapping is able to quantify myocardial fibrosis and can be used to quantify myocardial extracellular volume fraction (EVF), which changes when collagen content increases. We aimed to determine whether EVF quantification could detect subclinical left ven-

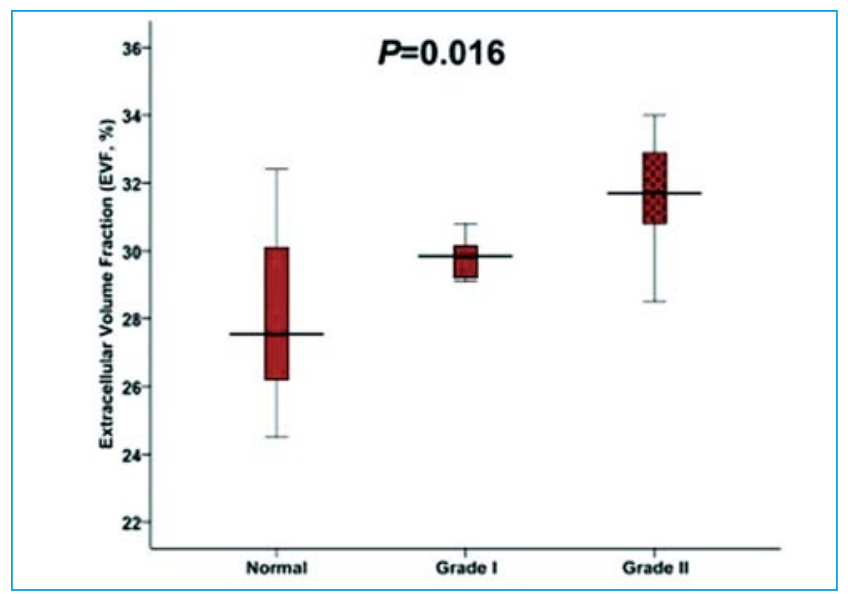

Figure 1. Extracellular volume fraction (EVF) according to the diastolic dysfunction pattern in patients with systemic sclerosis. tricular (LV) abnormalities and to investigate the relationship between EVF and subtle diastolic and systolic dysfunction.

Materials and Methods: T1 mapping and EVF quantification were prospectively performed in 33 consecutive SSc patients with normal echocardiography, no severe diastolic dysfunction, no pulmonary hypertension at rest and no LGE, as well as in 16 healthy controls. The study was approved by the local ethics committee and all subjects gave written informed consent.

Results: SSc patients had significantly higher global EVF $(P<0.0001)$ and higher local EVF for all basal and mid-ventricular LV segments $(P<0.01)$. Global EVF significantly correlated with left atrial volume $(\mathrm{P}<0.0001)$ and with the grade of diastolic dysfunction ( $P=0.016)$. Majority of SSc patients $(63 \%)$ had both a high global EVF and low global systolic circumferential strain.

Conclusions: EVF quantification can identify LV abnormalities at an early stage in SSc patients. These abnormalities may reflect increase in diffuse myocardial fibrosis and are associated with subtle diastolic and systolic LV dysfunction.

KEYWORDS: cardiac magnetic resonance, extracellular volume fraction, diastolic dysfunction, systemic sclerosis, myocardial fibrosis.

\section{Received: $20^{\text {th }}$ Mar 2013}

*Address for correspondence: Louis Pradel Hospital - Hospices Civils de Lyon, Université Claude Bernard Lyon, 28 avenue Doyen Lepine, 69677 Bron, France. Phone: +385-91-44-88 350

Fax: $+33-47-23-76-910$

E-mail: daniel@dlovric.net

\section{Literature}

1. Jellis C, Martin J, Narula J, Marwick TH. Assessment of nonischemic myocardial fibrosis. J Am Coll Cardiol. 2010;56:89-97.

2. Mewton N, Liu CY, Croisille P, Bluemke D, Lima JA. Assessment of myocardial fibrosis with cardiovascular magnetic resonance. J Am Coll Cardiol. 2011;57:891-903.

3. Tzelepis GE, Kelekis NL, Plastiras SC, et al. Pattern and distribution of myocardial fibrosis in systemic sclerosis: A delayed enhanced magnetic resonance imaging study. Arthritis Rheum. 2007;56:3827-36.

4. Messroghli DR, Radjenovic A, Kozerke S, Higgins DM, Sivananthan MU, Ridgway JP. Modified look-locker inversion recovery (molli) for high-resolution t1 mapping of the heart. Magn Reson Med. 2004;52:141-6.

5. Flett AS, Hayward MP, Ashworth MT, et al. Equilibrium contrast cardiovascular magnetic resonance for the measurement of diffuse myocardial fibrosis: Preliminary validation in humans. Circulation. 2010;122:138-44.

6. Kellman P, Wilson JR, Xue H, et al. Extracellular volume fraction mapping in the myocardium, part 2: initial clinical experience. J Cardiovasc Magn Reson. 2012 Sep 11;14:64. 